\title{
An Energy-aware Facilitation Framework for Scalable Social Internet of Vehicles
}

\author{
Abdulwahab Ali Almazroi, Muhammad Ahsan Qureshi \\ University of Jeddah, College of Computing and Information Technology at Khulais \\ Department of Information Technology \\ Jeddah, Saudi Arabia
}

\begin{abstract}
The Internet of Things (IoT) has eventually evolved into a more promising service provisioning paradigm, namely, Social Internet of Things (SIoT). Social Internet of Vehicles (SIoV) symbolizes a multitude of components from the existing Vehicular Ad-Hoc Networks (VANETs) such as OBUs, RSUs, and cloud devices that necessitate energy for proper functioning. It is speculated that the connected devices will surpass the 40 billion mark in the year 2022 in which the devices related to ITS will constitute a significant part. Therefore, the ever-increasing number of components increases the communication hopping that results in the immense escalation of energy consumption. However, the energy consumption at the object level increases due to individual communication, storage, and processing capabilities. The existing research in SIoV is focused on providing state-of-the-art services and applications; however, a significant goal of energy efficiency is largely ignored. Therefore, extensive research needs to be performed to come up with an energy-efficient framework for a scalable SIoV system to meet the future requirements of ITS. Consequently, this study proposed, simulated, and evaluated an energy-aware efficient deployment of RSUs scheme. The proposed scheme is based on network energy, data acquisition energy, and data processing energy. To achieve efficiency in terms of energy, traveling salesman problem with ant colony optimization algorithm are utilized. The experiments are performed in an urban scenario with different numbers of RSUs. The outcomes of the experiments exhibited promising results in energy gain and energy consumption having implications for society and consumers at large.
\end{abstract}

Keywords-Social Internet of Vehicles (SIoV); energy optimization; Travel Sales Person (TSP) problem; Ant Colony Optimization (ACO)

\section{INTRODUCTION}

VANETs [1] deploy vehicle to vehicle (V2V) and vehicle to infrastructure (V2I) communication to facilitate a wide range of applications Such as safety, navigation, routing, emergency healthcare, and infotainment. These applications are developed using VANETs due to the ability of VANETs to handle different topologies and management of continuously changing network densities. The effective utilization of VANETs is essential due to encouraging possible applications that includes both V2I and V2V communications. Both V2I and V2V communications are established in urban, suburban and highway environments with a wide range of specific topological features [2]. However, the advancements in network technology and the use of the internet allows the researchers to conceptualize the Internet of Vehicles (IoV) to develop applications that were not possible in traditional VANETs due to lack of all-time connectivity and unavailability of the internet [3]. IoV allows each network object to connect to the internet; hence, making it possible for potentially all OBUs, RSUs, and network users (passenger and drivers etc.) to share information resulting in a saleable communication environment.

Over time, IOV evolved in Social Internet of Vehicles (SIoV) in which the network objects can share information about common interests such as road conditions, traffic information, available parking spaces and resource sharing [4]. The network objects in SIoV are not limited to RSUs and OBUs, as the network may include users pedestrians, drivers, and passengers. Therefore, the vehicles in SIoV maintain social relations among them and other network objects depending upon the mutual interests such as real-time traffic conditions on a particular road segment. Due to highly dynamic nature of SIoV, the network objects join and leave the networks very fast and the connections are based on the same travel route and similar configurations etc. Therefore, the objects in the SIoV are equipped with communication, storage and processing capabilities, hence, making them smart objects.

The dynamic nature of the network, real-time information sharing, and the smart nature of the network objects in SIoV causes an immense generation of data that needs to be communicated, processed and stored [5]. Therefore, the energy consumption by individual network objects increases due to individual communication, storage, and processing capabilities [6]. Furthermore, the decentralized nature of the network does not impose any restriction on redundant data generation, dissemination, and storage that eventually results in wastage of energy and resources [7]. The existing research in the SIoV does not focus on energy-efficiency framework for SIoV systems considerably.

The technological advancements in recent years have led to increased carbon dioxide emissions. In the domain of IoT, efficient utilization of energy is needed to enable a greener IoT environment. The major focus for a greener IoT environment is on the efficient deployment of the fixed network objects to reduce the energy cost [18]. Although, the enabling technologies for greener IoT environment such as green sensing networks and green tags exists, however, there is a lack of a framework that considers all the possibilities to optimally utilize power, especially in SIoV domain. The green IoT itself is considered as the enabling technology for scalable and sustainable development and has multiple benefits such as 
environmental protection and customer satisfaction [19]. Power consumption in IoT systems is modeled at multiple levels such as measuring network energy, estimating data acquisition energy and modeling data processing energy [20]. Furthermore, multiple wireless energy harvesting techniques are available for empowering the self-sustained network with potentially an unlimited supply of power [21]. Therefore, the ground is all set to propose a novel energy-efficient framework for SIoV with the help of enabling technologies, energy prediction, and measurement models.

Therefore, the aim of this work is to propose an energyefficient framework to efficiently utilize the fixed network objects to reduce the energy cost for SIoV. To achieve the aim of the study, the following research objectives are proposed:

Objective 1: To study state-of-the-art in energy efficiency and SIoV.

Objective 2: To propose an energy efficiency framework suitable for SIoV systems.

Objective 3: To evaluate the proposed framework in terms of optimal utilization of energy.

Objective 4: To compare the proposed framework with existing solution(s).

The rest of the paper is organized as follows: Section 2 presents state-of-the-art studies related to SIoV and energy efficiency. This is then followed by Section 3, which proposes an energy-efficient framework for SIoV. The experimental setup and results are presented in Section 3 and 4, respectively. The last section concludes the study, discusses future directions, and limitations of the study.

\section{RELATED WORK}

Traditional IoV frameworks provide safety, infotainment and traffic efficiency related information that is shared among other vehicles and infrastructure [8]. Some of the existing studies also deal with social networks [7, 9]. Firstly, a few studies related to vehicular communication and SIoV are presented to show the immense amount of data that is generated and disseminated using the applications and services. Secondly, the major work related to energy efficiency in the domain of IoV and SIoV is described in this section. The current focus of energy efficiency is in three dimensions, which are, Internet, tags, and sensors. Fig. 1 shows the enabling technologies for green IoT.

The traffic congestion and road accidents are caused by the number of ever-increasing vehicles on the road. SIoV has the potential to resolve these issues by providing services and applications ranging from infotainment to security. According to authors in [9], SIoV consists of six components: tNote message, OBUs, RSUs, Home Base Units (HBU), tNote cloud and User Interface. A framework for social information sharing among vehicles is provided, however, redundant data generation is not handled which is the main cause of excessive energy consumption in the SIoV domain. An attempt [17] was made to reduce the overall power consumption of IoT system by deploying unmanned aerial vehicles (UAVs) that serve as base stations. The UAVs were used to collect data from IoT devices for reliable uplink communications. The study also presents a framework for the optimal placement of UAVs. However, this framework is not suitable for SIoV implementation because the nature of the vehicular environment varies significantly in terms of vehicular speed and road topology.

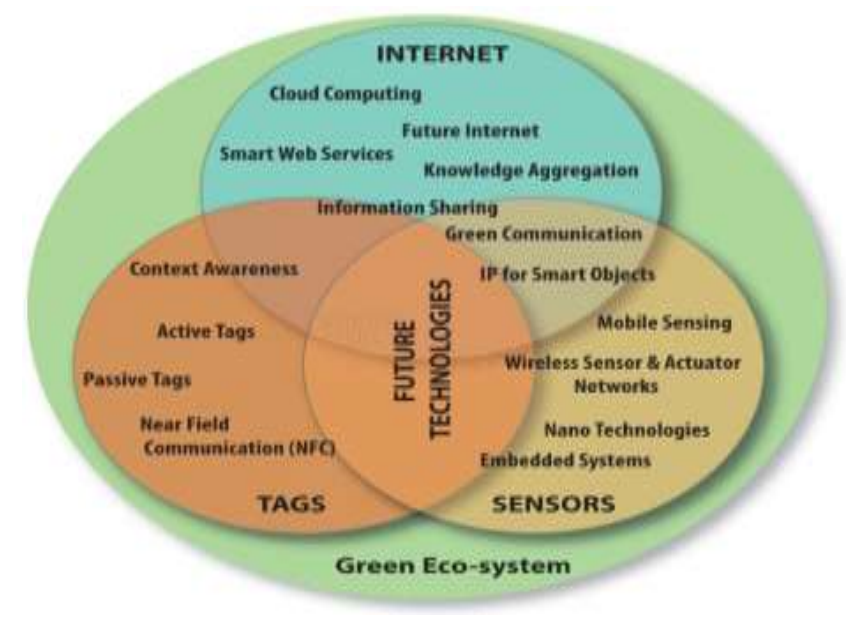

Fig. 1. Enabling Technologies for Green IoT [18].

Another model for VANETs exists in the literature which is referred to as VANET-cloud [10] that provides multiple services, applications and digital platforms to users at a relatively low cost [11]. Further, this model claims to improve safety by exchanging traffic data to vehicles and other network objects for the appropriate action in multiple traffic situations. Moreover, this model offers revenues to the drivers by sharing their onboard computing resources with other network objects. Modern autonomous vehicles may become the future of urban transportation for a relatively safer experience [12]. These autonomous vehicles are equipped with sensors that generate large amounts of data. The road is instrumented with components, RFID tags, and embedded microcontrollers. Vehicular cloud provides a communication and computing environment in order to provide services for autonomous vehicles. Hence the amount of data is constantly generated and processed.

Social vehicular network (SVN) technique consists of Vehicular Cloud (VC), RSU, and Internet Cloud (IC) components [8,13]. Vehicular cloud establishes a connection with RSU. VC communicates with IC via RSU. However, no attention is given to minimizing the number of messages exchanged among the network objects. A Smart-Eye technique for a safer driving experience is presented in [14]. The information is shared in multiple formats ranging from text, video and coordinates. However, this technique lacks security and privacy along with the redundant data generation and dissemination causing the wastage of energy. Further, the existing studies advocate the use of fog computing to overcome the deficiency of central data processing related to traditional cloud computing by offloading the selected tasks on the edge of the network $[15,16]$. Hence, it reduces the communication cost; thereby resulting in the consequent reduction in the communication energy usage. However, in the IoV domain, the decentralized nature of fog computing may cause redundant 
data to be generated and processed eventually. Therefore, without a framework for energy efficiency, the mere use of fog computing cannot guarantee the optimal use of power and energy in communication and processing.

\section{PROPOSED FRAMEWORK}

An energy-aware facilitation framework for SIoV is proposed in the current study to efficiently utilize the fixed network objects to reduce the energy cost. Specifically, the proposed framework offers energy-aware efficient deployment of RSUs based upon three energy consumption types: (i) network energy, (ii) data acquisition energy, and (iii) data processing energy. The proposed framework is depicted in Fig. 2. The input to the system is the network traffic and the output is energy-aware deployment of RSUs. In the framework, the resource request is received to RSUs from network traffic. After that, network energy is measured. Subsequently, data acquisition energy is estimated and data processing energy is modeled. Based on these three types of energies, intelligent energy-aware deployment of RSUs is proposed resulting in lower energy consumption, thus dropping the overall energy cost.

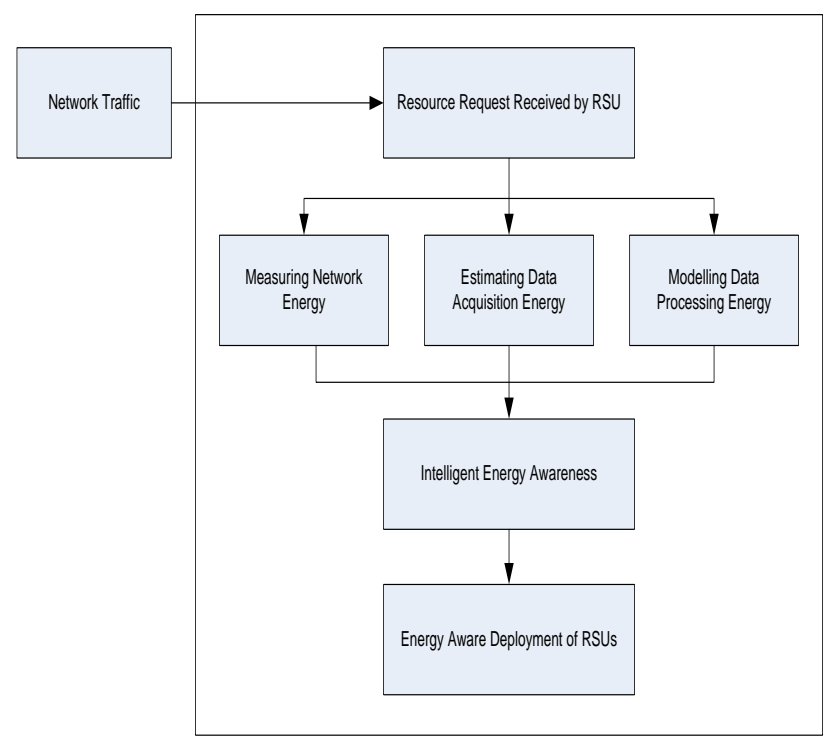

Fig. 2. Proposed Energy Facilitation Framework for SIoV.

The intelligent energy awareness in this framework can be obtained by using state-of-the-art artificial intelligence and machine learning techniques. One of the candidates in this regard is the solution of the classical traveling salesperson (TSP) problem based on the ant colony optimization algorithm (ACO) [22-25]. In the first step, a path with optimal energy consumption is calculated using the shortest Hamiltonian cycle. The second step consists of the ant colony optimization application. The two steps are explained below:

Given a collection of RSUs, the objective is to find the shortest Hamiltonian cycle connecting all the RSUs in a given set. So that, the acknowledgment should also be received. This is because the required resource might be present at any node which is connected to a particular RSU. This can be mapped as the classic traveling saleperson problem. The collection of the RSUs can be mapped onto a collection of cities in TSP. The path between RSUs having optimal energy consumption can be regarded as the shortest Hamiltonian cycle. The mathematical model of TSP in terms of SIoV is presented below:

Consider a weighted graph $\mathrm{G}=(\mathrm{R}, \mathrm{C})$ where $\mathrm{R}$ is the set of $\mathrm{n}$ RSUs and C represents the connection linking the RSUs.

Each connection $(i, j) \in C$ is associated with $E_{i j}$ that represents the energy cost between RSUs i,j.

However, SIoV is a highly dynamic environment, therefore, the energy cost $\mathrm{E}_{\mathrm{ij}}$ is bound to be changed. So,

$E(T)=\left\{E_{i j}(T)\right\} n * n$

Here, $\mathrm{E}()$ is the energy cost and $\mathrm{T}$ is the period in which there is no change in the energy cost.

$\mathrm{T}=\mathrm{t} / \mathrm{f}$, here $\mathrm{t}$ is the time and $\mathrm{f}$ encodes the frequency of the occurrence of the change.

In the current study, the famous ant colony optimization algorithm is used for optimization purpose. In the field of computer science, this algorithm is used to find the optimal path through a graph by using the probabilistic technique. The ACO can be applied to optimization problems to find the best path on a weighted graph. In SIOV terms, RSUs can be regarded as the nodes of a weighted graph whereas the edges between the nodes encode the energy consumption. Initially, ants are placed at RSUs randomly; the probability with which an ant " $k$ " chases the next RSUs is given by the following Equation:

$\rho_{i j}^{k}(t)=\frac{\left[\tau_{i j}(t)\right]^{\alpha} \cdot\left[\eta_{i j}\right]^{\beta}}{\sum_{l \in j_{i}^{k}}\left[\tau_{i l}(t)\right]^{\alpha} \cdot\left[\eta_{i l}\right]^{\beta}}$

In the above equation $\tau_{\mathrm{ij}}$ is the pheromone value deposited for a transition from one state to another, and $\eta_{i j}$ is the reciprocal of $E_{i j}(T)$ depicting the visibility, the parameter $\alpha$ is related with pheromone and $\beta$ is associated with visibility information.

The pheromone value $\tau_{\mathrm{ij}}$ is updated using the following equation:

$\tau_{x y} \leftarrow(1-\rho) \tau_{x y}+\sum_{k}^{m} \Delta \tau_{x y}^{k}$

\section{EXPERIMENTAL SETUP}

Simulation of urban mobility (SUMO) tool is utilized in the work for traffic simulation as it is widely used for traffic traces generation and simulating road conditions in different scenarios. The focus of the study is urban scenario instead of highway scenario. A $9 \mathrm{Km} 2$ area is selected for modeling urban traffic as shown in Fig. 3. Energy consumption values are randomly assigned to each RSU dynamically in the selected area based on their processing requirements. The Ant Colony optimization is implemented using Python 3.9. The experimental setup is depicted in Fig. 4. For the experimental purpose, the number of RSUs varies in the selected area, that is, five RSUs are incremented in every experiment, and therefore, the experimental results are attained with 5, 10, 20 and 25 RSUs. 


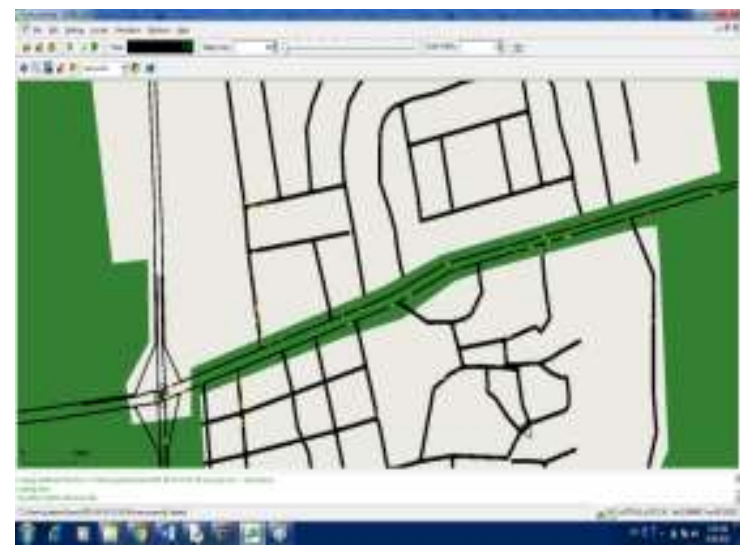

Fig. 3. Urban Scenario as Represented in SUMO.

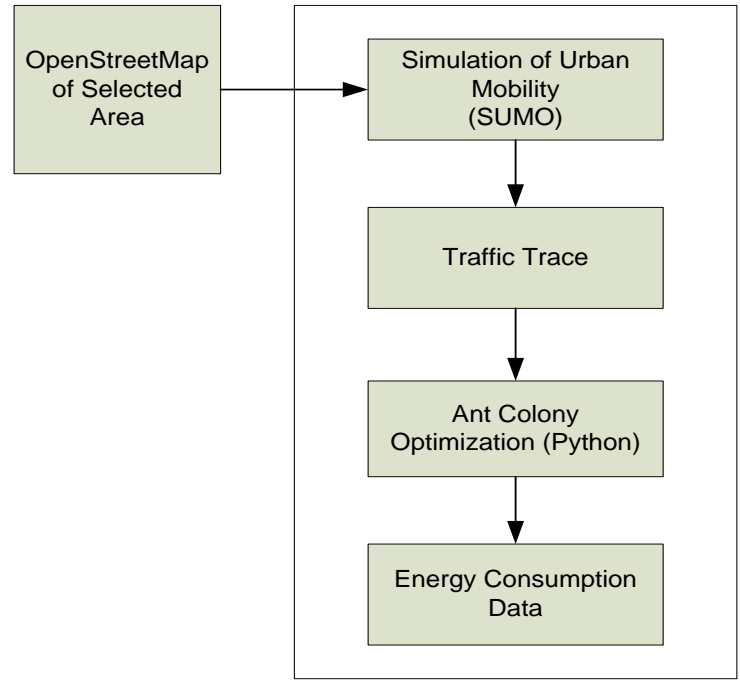

Fig. 4. Experimental Setup.

\section{EXPERIMENTAL RESULTS}

This section presents the results of the experiments conducted by using the proposed framework. The energy consumption is calculated for different numbers of RSUS. Specifically, the energy consumed is measured with $5,10,15$, 20, and 25 RSUs as depicted in Fig. 5. The experimental results support that the proposed framework saves energy in all experiments with different number of RSUs. The energy consumption with $5,10,15,20,25$ RSUs is less with ant colony optimization as seen in Fig. 5. Therefore, it is concluded that optimization techniques can results in less energy consumption, thus, minimizing the overall cost.

With the increased number of RSUs (and hence communicating vehicles), the relative energy consumption is reduced. However, other optimization techniques may also be used to further validate the results of this study.

Fig. 6 exhibited the energy gain by conducting the experiments with energy-aware facilitation framework for SIoV. The considerable energy gain is accomplished with different numbers of RSUs. The highest energy gain with a value of 7 is achieved with 25 RSUs which is remarkable. Therefore, it can be inferred that the energy gain can be increased by adding more RSUs. However, it is also interesting to note that the second highest gain in energy (a value of 5) is a result of five RSUs. Therefore, we can say that the best energy gain is achieved with the deployment of minimum and maximum RSUs. On the other hand, a considerable energy gain of 3, 4, and 4 values is observed with 10,15 , and 20 RSUs, respectively. Therefore, the results of the study confirmed the energy saving by deploying the proposed energy-aware framework.

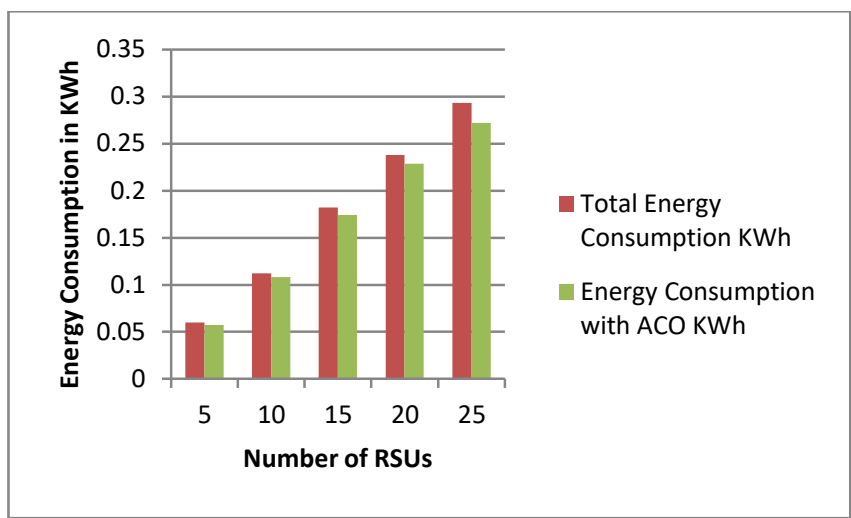

Fig. 5. Energy Consumed with different Number of RSUs.

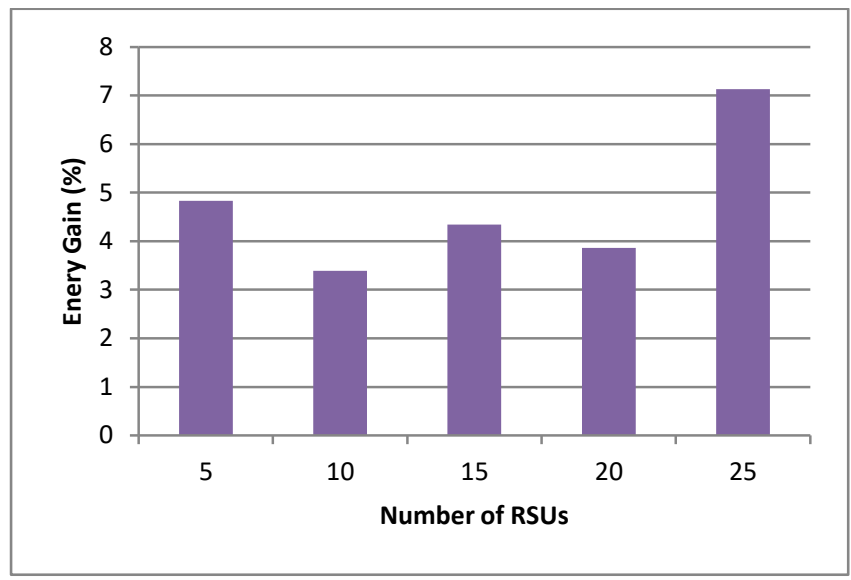

Fig. 6. Energy Gain.

\section{RESEARCH IMPLICATIONS}

The expected outcomes of the study have implications for the scientific community, society and consumers. The implications of expected results in scientific community perceptive are many folds. First, the expected results can be utilized for the scientific community to design and develop new applications, standards and protocols based upon the recommendations of the proposed energy-efficient framework. Second, the new applications, standards and protocols in SIoV domains will be evaluated in terms of the amount of energy they consume. Third, the decision of whether to adopt a new application for commercial use may depend upon the evaluation from the proposed framework causing only energyefficient applications to be available commercially resulting in less energy consumed in SIoV. Fourthly, redundant information generation, dissemination, and storage will be reduced, thus, saving cost as well as energy. In commercial use, energy saving and cost reduction play an important role in escalating the revenue of an organization. The main focus of 
any business organization is to maximize revenue. Therefore, the proposed framework may have a huge impact on business organizations that want to accelerate their profit by reducing cost and energy consumption. Lastly, due to global energy crises, many countries, like Saudi Arabia will not rely on fossil fuel and currently looking for energy-efficient solutions. Therefore, the energy-efficiency is required as it has a huge impact on general public as well as the corporate section. In short, the greener technological advancements can be advantageous for both consumers and corporate and participating in the betterment of society at large.

\section{CONCLUSION, FUTURE WORK AND LIMITATIONS}

The innovation in network technology and IoV resulted in the conceptualization of SIoV. There are multiple objects including, RSUs, OBUS, vehicles, drivers, and passengers that have a social connection with each other for sharing information related to traffic condition and density, parking spaces and other resources in SIoV. These objects have communication, storage, and processing competencies to maintain social connections. The smart nature of network objects, real-time information sharing, and dynamic nature of network, redundant data generation resulted in the production of enormous data that require energy and resources for processing, storage, and communication. Therefore, the current work provides an energy-efficient framework to reduce the energy cost in SIoV. The proposed framework is based on network energy, data acquisition energy, and data modeling energy. The classical travel salesperson problem and ant colony optimization algorithm are applied for calculation of path requiring less energy from source to destination. The proposed framework is simulated in the urban setting within a 9 KM2 area. The experiments conducted with 5, 10, 15, 20, and 25 RSUs supporting significantly the energy gain achieved through the proposed method. Further, the total energy consumption is less by applying the proposed framework as highlighted by the experimental results. The study has some limitations. First, the proposed solution is evaluated on small scale by developing a real-world testbed application. Second, only simulation results will be presented. Future directions of this study include real-world evaluation of the framework with a higher number of RSUs.

\section{ACKNOWLEDGMENT}

This work was funded by the Deanship of Scientific Research (DSR), University of Jeddah, Jeddah, under grant No. ((UJ-02-066-DR). The authors, therefore, acknowledge with thanks DSR technical and financial support.

\section{REFERENCES}

[1] M. A. Qureshi, et al., "A survey on obstacle modeling patterns in radio propagation models for vehicular ad hoc networks," Arabian Journal for Science and Engineering, vol. 40, pp. 1385-1407, 2015.

[2] M. A. Qureshi, et al., "A lightweight radio propagation model for vehicular communication in road tunnels," PloS one, vol. 11, p. e0152727, 2016.

[3] O. Kaiwartya, et al., "Internet of vehicles: Motivation, layered architecture, network model, challenges, and future aspects," IEEE access, vol. 4, pp. 5356-5373, 2016.

[4] T. A. Butt, et al., "Social Internet of Vehicles: Architecture and enabling technologies," Computers \& Electrical Engineering, vol. 69, pp. 68-84, 2018.
[5] L. Maglaras, et al., "Social internet of vehicles for smart cities," Journal of Sensor and Actuator Networks, vol. 5, p. 3, 2016.

[6] G. Xiong, et al., "Cyber-physical-social system in intelligent transportation," IEEE/CAA Journal of Automatica Sinica, vol. 2, pp. 320-333, 2015.

[7] Z. Zhou, et al., "Social big-data-based content dissemination in Internet of vehicles," IEEE Transactions on Industrial Informatics, vol. 14, pp. 768-777, 2018.

[8] D. Kwak, et al., "Seeing is believing: Sharing real-time visual traffic information via vehicular clouds," IEEE access, vol. 4, pp. 3617-3631, 2016.

[9] K. M. Alam, et al., "Toward social internet of vehicles: Concept, architecture, and applications," IEEE access, vol. 3, pp. 343-357, 2015.

[10] S. Bitam, et al., "VANET-cloud: a generic cloud computing model for vehicular Ad Hoc networks," IEEE Wireless Communications, vol. 22, pp. 96-102, 2015.

[11] I. Ahmad, et al., "The role of vehicular cloud computing in road traffic management: a survey," in International Conference on Future Intelligent Vehicular Technologies, 2016, pp. 123-131.

[12] E.-K. Lee, et al., "Internet of Vehicles: From intelligent grid to autonomous cars and vehicular fogs," International Journal of Distributed Sensor Networks, vol. 12, p. 1550147716665500, 2016.

[13] M. Aloqaily, et al., "An auction-driven multi-objective provisioning framework in a vehicular cloud," in 2015 IEEE Globecom Workshops (GC Wkshps), 2015, pp. 1-6.

[14] D. Singh and M. Singh, "Internet of vehicles for smart and safe driving," in 2015 International Conference on Connected Vehicles and Expo (ICCVE), 2015, pp. 328-329.

[15] W. Zhang, et al., "Cooperative fog computing for dealing with big data in the internet of vehicles: Architecture and hierarchical resource management," IEEE Communications Magazine, vol. 55, pp. 60-67, 2017.

[16] K. Zhang, et al., "Mobile edge computing and networking for green and low-latency Internet of Things," IEEE Communications Magazine, vol. 56, pp. 39-45, 2018.

[17] M. Mozaffari, et al., "Mobile unmanned aerial vehicles (UAVs) for energy-efficient internet of things communications," IEEE Transactions on Wireless Communications, vol. 16, pp. 7574-7589, 2017.

[18] F. K. Shaikh, et al., "Enabling technologies for green internet of things," IEEE Systems Journal, vol. 11, pp. 983-994, 2017.

[19] M. Maksimovic, "Greening the future: Green Internet of Things (G-IoT) as a key technological enabler of sustainable development," in Internet of things and big data analytics toward next-generation intelligence, ed: Springer, 2018, pp. 283-313.

[20] B. Martinez, et al., "The power of models: Modeling power consumption for IoT devices," IEEE Sensors Journal, vol. 15, pp. 57775789, 2015.

[21] P. Kamalinejad, et al., "Wireless energy harvesting for the Internet of Things," IEEE Communications Magazine, vol. 53, pp. 102-108, 2015.

[22] Aadil, F., Ahsan, W., Rehman, Z.U., Shah, P.A., Rho, S. and Mehmood, I., 2018. Clustering algorithm for internet of vehicles (IoV) based on dragonfly optimizer (CAVDO). The Journal of Supercomputing, 74(9), pp. $4542-4567$.

[23] Ebadinezhad, S., Dereboylu, Z. and Ever, E., 2019. Clustering-based modified ant colony optimizer for internet of vehicles (CACOIOV). Sustainability, 11(9), p.2624.

[24] Zhang, J., Liu, B., Zhang, W. and Jiang, D., 2020, August. An IoV Route Planning Service Based on LEO Constellation Satellites. In International Conference on Simulation Tools and Techniques (pp. 194203). Springer, Cham.

[25] Kumar, S., Solanki, V.K., Choudhary, S.K., Selamat, A. and González Crespo, R., 2020. Comparative Study on Ant Colony Optimization (ACO) and K-Means Clustering Approaches for Jobs Scheduling and Energy Optimization Model in Internet of Things (IoT). International Journal of Interactive Multimedia \& Artificial Intelligence, 6(1). 